\title{
A POF Model for Short Fiber Segments in Avionics Applications
}

\author{
Natali Pujols ${ }^{*+}, M^{a}$ Ángeles Losada ${ }^{* *}$, Javier Mateo $^{* *}$, Alicia López $^{* *}$, Dwight Richards*, \\ N. Madamopoulos ${ }^{+}$, Senior Member, IEEE, and N. Antoniades", Senior Member, IEEE \\ * College of Staten Island, The City University of New York, Staten Island, NY 10314, USA \\ **GTF, Aragón Institute of Engineering Research (i3A), Universidad de Zaragoza, Zaragoza, Spain \\ +Department of Electrical Engineering, The City College of CUNY, New York, NY, 10031, USA \\ and Department of Aeronautical Sciences, Hellenic Air Force Academy, Dekeleia, 13671, Greece \\ Tel: +1 7189822824, email: npujols00@ citymail.cuny.edu
}

\begin{abstract}
Plastic Optical Fibers (POF) have now been a well-established media for transporting high-speed at low cost in short distance communications systems, and avionics is the latest segment where these fibers are becoming an important factor. In fact, the POF's flexibility and ease of installation make this fiber a great option for the replacement by fiber of some of the existing aircraft data links. We propose a new model for short fiber links that are suitable for plane's systems that involve a lot of connectors; the model is built around two important properties, namely, power transfer among modes and attenuation. The model expands on our previous work on the subject and is based on detailed experimental measurements for various fiber types and lengths.
\end{abstract}

Keywords: Plastic optical fibres, optical networks, simulation software, data communications, avionics.

\section{INTRODUCTION}

Plastic optical fiber's (POF) high attenuation has limited POF to short-range communication systems. Although glass fiber can achieve long distance transmissions, its small and fragile core makes the fiber susceptible to breakage during the installation process and requires precise training for handling, testing and maintenance. POF's large core, high numerical aperture and flexibility, among other things, reduces the hardships in the fiber's handling, testing and maintenance, making the fiber attractive for home, automotive and recently commercial avionic applications [1-2]. The need of simulation tools to study emerging POF communication systems and applications has motivated us to develop a POF model compatible with commercial simulation software like OptSim [2]. Before this work, our model had been tested using relatively long fibers. In the case of avionic applications, which is our targeted application space, the number of connectors used in a typical path, which is fairly high, results in a significant shortening of the fiber's individual lengths thus making the case for a reliable model adaption for short POF segments.

This article pertains to a new developed POF model that is best described using the modal power distribution in a multimode fiber involving Gloge's power flow equation [3] and is intended for modelling short fiber segments in an avionics system platform. The new model is experimentally verified and is built around two important properties, namely, power transfer among modes and attenuation. The power transfer among modes, or diffusion, and the attenuation are described using functions solely dependent on the propagation angle. To adapt the model for systems using short POF fiber, we propose simulating the attenuation and diffusion functions using splines instead of the proposed functions in [4]. To find the splines that better describe the fiber's power distribution, the fitting method used compares the experimental and the modelled angular power distribution matrices at a particular length of the fiber. This process is repeated using different fiber lengths to explore the function's variability with length. Using three different fibers with lengths ranging from $2.5 \mathrm{~m}$ to $15 \mathrm{~m}$, we present their corresponding attenuation and diffusion functions as well as the error between the experimental and the fitted power distribution matrices.

This paper is organized as follows: in section 2 the matrix model for POF fiber is presented as a starting point. Section 3 covers the experiment setup used to obtain the far field pattern (FFP) radial profiles that leads to the new model. Section 4 includes a description of the fitting process used and an analysis of the results obtained. Lastly, section 5 will have our summarized conclusions.

\section{MATRIX MODEL FOR PLASTIC OPTICAL FIBRE AND PASSIVE COMPONENTS}

The power flow for a multimode fiber can be described using Gloge's power flow equation. Various methods based on Gloge's power flow equation have been used to describe the power distribution of POF fibers [5]-[6]. In our case, a matrix approach has been developed to represent the power distribution of a POF fiber. For this approach if the power distribution at length $z_{1}, \mathbf{p}\left(z_{1}, \omega\right)$, is known then the power distribution at length $z_{2}$ is the product of $\mathbf{p}\left(z_{2}, \omega\right)$ and the fiber characteristic matrix $\mathbf{S}$, namely.

$$
\mathbf{p}\left(z_{2}, \omega\right)=\mathbf{p}\left(z_{1}, \omega\right) \cdot \mathbf{S}\left(\omega, z_{1}, z_{2}\right)=\mathbf{p}\left(z_{1}, \omega\right) \cdot(\mathbf{A}(\omega)+\mathbf{D})^{\left(z_{2}-z_{1}\right) / \Delta z}
$$

The matrix $\mathbf{A}(\omega)$ is a diagonal matrix that describes the power propagation in the fiber in the absence of diffusion. The matrix $\mathbf{D}$ is a tridiagonal matrix that describes the diffusion along the fiber. The objective of this 
paper is to find the spatial representation of $\mathbf{A}$ and $\mathbf{D}$. Therefore we can set $\omega$ equal to zero, in which case the resulting matrices are defined as follows:

$$
\begin{gathered}
A_{k, k}=\exp (\Delta z \cdot \alpha(k \cdot \Delta \theta)) \\
D_{k, k-1}=\left(d(k \cdot \Delta \theta)-\frac{1}{2} \frac{d(k \cdot \Delta \theta)}{k}-\frac{1}{2} d^{\prime}(k \cdot \Delta \theta) \cdot \Delta \theta\right) \frac{\Delta z}{\Delta \theta^{2}} \\
D_{k, k}=-2 d(k \cdot \Delta \theta) \frac{\Delta z}{\Delta \theta^{2}} \\
D_{k, k+1}=\left(d(k \cdot \Delta \theta)+\frac{1}{2} \frac{d(k \cdot \Delta \theta)}{k}+\frac{1}{2} d^{\prime}(k \cdot \Delta \theta) \cdot \Delta \theta\right) \frac{\Delta z}{\Delta \theta^{2}}
\end{gathered}
$$

where $k \cdot \Delta \theta=\theta$ is the discretized propagation angle, $k$ is a real positive integer, $\Delta z$ is the length step, $\alpha(\theta)$ and $d(\theta)$ describe the angular attenuation and diffusion respectively. For a more detailed explanation on the previous equations, including the boundary conditions for matrix $\mathbf{D}$, the reader is referred to [7]. In order to make this model suitable for simulating systems using POF fibers it is imperative to find the $\alpha(\theta)$ and $d(\theta)$ functions that will describe the fiber at any length. In a previous work [4], we have assigned specific functions for $\alpha(\theta)$ and $d(\theta)$ for the case when the angular power distribution has achieved steady state. The steady state occurs at long distances, therefore the method in [4] is more suitable for long fibers. To expand the model's applicability to other lengths which, as we mentioned before, are suitable for avionics applications, we will assume that $\alpha(\theta)$ and $d(\theta)$ are unknown and independent. The fitting approach to find these unknown functions needs to be performed for different lengths in order to determine any dependence on the fiber length. The insights on the latter statement will be discussed in more details in Section 4.

\section{EXPERIMENT SETUP TO ACQUIRE FFP IMAGES AND RADIAL PROFILES}

To obtain the power distribution of a particular fiber, FFPs were measured at different input angles. The experimental procedure to obtain a FFP starts with the transmission of light using a collimated He-Ne laser of $635 \mathrm{~nm}$. The light is then injected into the fiber which is placed on a rotatory base aligned with the laser beam. The base then rotates from $-40^{\circ}$ to $+40^{\circ}$ from the laser propagation axis at a step of $1^{\circ}$. This angle at which the fiber axis deviates from the laser propagation axis is defined as the input angle. The FFP image is then reflected on a white screen located at $7.5 \mathrm{~cm}$ from the fiber output end. An image of the FFP is taken using a 12 bit monochrome cooled camera QICAM FAST 1394 [8]. To reduce the speckle noise a mobile phone vibrator is placed right before the fiber output end.

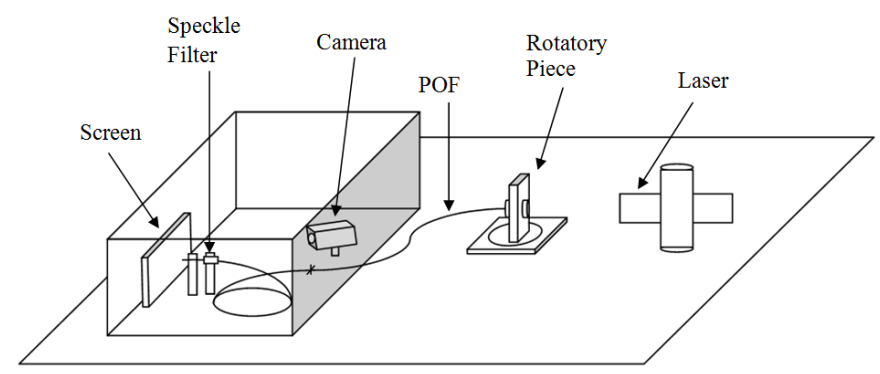

Figure 1. Experiment setup

From the measured FFPs, radial profiles were extracted by averaging the values of all the pixels at a given distance from the centroid of the FFP [8]. The power distribution is a matrix whose columns correspond to the radial profiles obtained for all the input angles as functions of the output angle. The output angle is no other than the angle of incidence of the light ray striking the screen. Three different fibers were used to test the described model. These PMMA fibers of $1 \mathrm{~mm}$ diameter are: ESKA PREMIER GH4001 (GH) from Mitsubishi, PGUFB100 (PGU) from Toray, and HFBR-RUS100 (HFB) from Agilent. The GH and PGU fibers have a numerical aperture (NA) of 0.5 , for an inner critical angle of $19.5^{\circ}$, and a $0.15 \mathrm{~dB} / \mathrm{m}$ nominal attenuation. For the HFB fibers the NA is 0.47 , for an inner critical angle of $18.5^{\circ}$, and the nominal attenuation is $0.22 \mathrm{~dB} / \mathrm{m}$. The power distribution matrix was obtained for each fiber starting with a length of $15 \mathrm{~m}$, then by keeping the injection end fixed, the power distribution is obtained for the same fiber shortened to $10 \mathrm{~m}, 5 \mathrm{~m}$ and $2.5 \mathrm{~m}$.

\section{FITTING METHOD AND RESULTS}

To find the matrix $\mathbf{A}$ and $\mathbf{D}$ that represents a particular POF fiber, we must find $\alpha(\theta)$ and $d(\theta)$ as described above. Due to the uncertainty on the curve shapes for $\alpha(\theta)$ and $d(\theta)$, it is imperative to select a method that will allow us to fit the characteristic matrix without assuming any specific functions describing their respective 
curves. To achieve this, $\alpha(\theta)$ and $d(\theta)$ are represented as splines. The fitting process is then performed using the fmincon() function in Matlab to find the splines that will minimize the following cost function:

$$
c f=\frac{\left\|\mathbf{p}_{\text {exp }}-\mathbf{p}_{\text {fit }}\right\|}{\left\|\mathbf{p}_{\text {exp }}\right\|}
$$

where $\mathbf{p}_{\text {exp }}$ is the experimental power distribution matrix at length $z_{2}$ and $\mathbf{p}_{f i t}$ is the power distribution matrix at length $z_{2}$ using the fitting results. Selecting an adequate cost function plays an important part in the fitting process. In addition to the cost function there are other factors to be considered, these are: initial values, boundary limits and the number of spline segments for each function. For the fitting process the initial values for the $\alpha(\theta)$ function are obtained directly from the measurements by following the procedure in [9]. By choosing these values it is possible to reduce the level of uncertainty regarding the curve's shape. As a result the minimization process yields smaller errors when compared to random initial values. In the case of $d(\theta)$ there is no approximation to estimate the curve as for $\alpha(\theta)$, therefore random initial values are selected. The boundary limits, namely the upper and lower boundaries, must keep the fitted curve within a relatively narrow range of positive values. This will prevent having curves with negative values, which have no physical sense for the attenuation nor the diffusion, in addition to obtaining smoother spline curves. Regarding the amount of segments used to approximate the $\alpha(\theta)$ and $d(\theta)$ functions, we have selected splines with four and seven segments respectively. The number of segments that was used has been selected based on the changes in the overall error.

To find the values that better describe the fiber, we have selected different pairs of $z_{2}$ and $z_{1}$, namely, $5 \mathrm{~m}$ and $2.5 \mathrm{~m}, 10 \mathrm{~m}$ and $2.5 \mathrm{~m}, 15 \mathrm{~m}$ and $2.5 \mathrm{~m}, 10 \mathrm{~m}$ and $5 \mathrm{~m}, 15 \mathrm{~m}$ and $5 \mathrm{~m}$, and $15 \mathrm{~m}$ and $10 \mathrm{~m}$. The main idea is to use the results obtained from each pair and try to reproduce the experimental power distribution for each fiber of length $z_{2}$. This will allow us to determine which pair yields the smallest error. Fig. 2, presents the error obtained, in percentage form, when using the average results for each pair of lengths. Average results are obtained by averaging the fitting results for each fiber length pair. In this case we used GH fibers with lengths up to $15 \mathrm{~m}$ while for the PGU and HFB fibers we used up to $10 \mathrm{~m}$. We also take the positive and negative injection angles from each fiber's power distribution matrix as separate measurements.
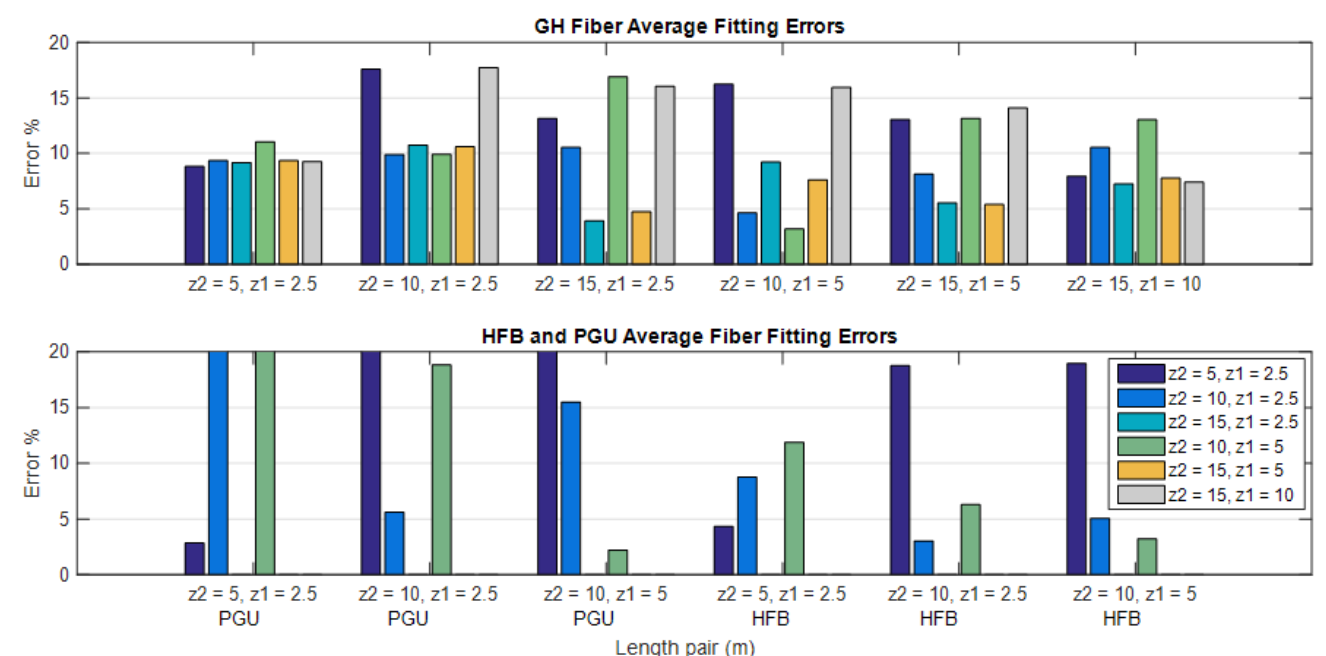

Figure 2: Average error for modeling the GH, HFB, PGU fiber using different length pairs.

For a particular length pair the error is smaller when using the results corresponding to the same length pair. In other words if it is intended to model $15 \mathrm{~m}$ of fiber using length $z_{1}$ of $5 \mathrm{~m}$, we need to use the $15 \mathrm{~m}$ and $5 \mathrm{~m}$ average results. Although for some cases the smallest error is achieved using a different pair of lengths, when considering the consistency in the results, using the results for the same pair of lengths seems more appropriate. In Fig. 3, the attenuation and diffusion functions are presented for the GH, PGU, and HFB fibers using different length combinations. Results for pair of lengths with $z_{1}=2.5 \mathrm{~m}$ should be treated carefully since the fitting results for these pairs were rather inconsistent. In this case the fiber is not long enough to start exhibiting any pattern since, at $2.5 \mathrm{~m}$, the modes in the fiber have not travelled long enough to produce a steady trend in the attenuation and diffusion patterns. This is most noticeable when using two short fibers. As we can observe in Fig. 3 , the $5 \mathrm{~m}$ and $2.5 \mathrm{~m}$ length pair of each fiber deviates significantly from the pattern followed by the rest of the fiber length pairs. To further improve the measurements it is necessary to take into consideration the following factors: alignment, fiber termination, and curvatures. In our experiments these factors have proven to influence the intensities and symmetry of the angular power distribution. Although, the details regarding these factors are beyond the scope of this article, when performing the experiment in section 3 it is imperative to address these factors before starting the data acquisition. 

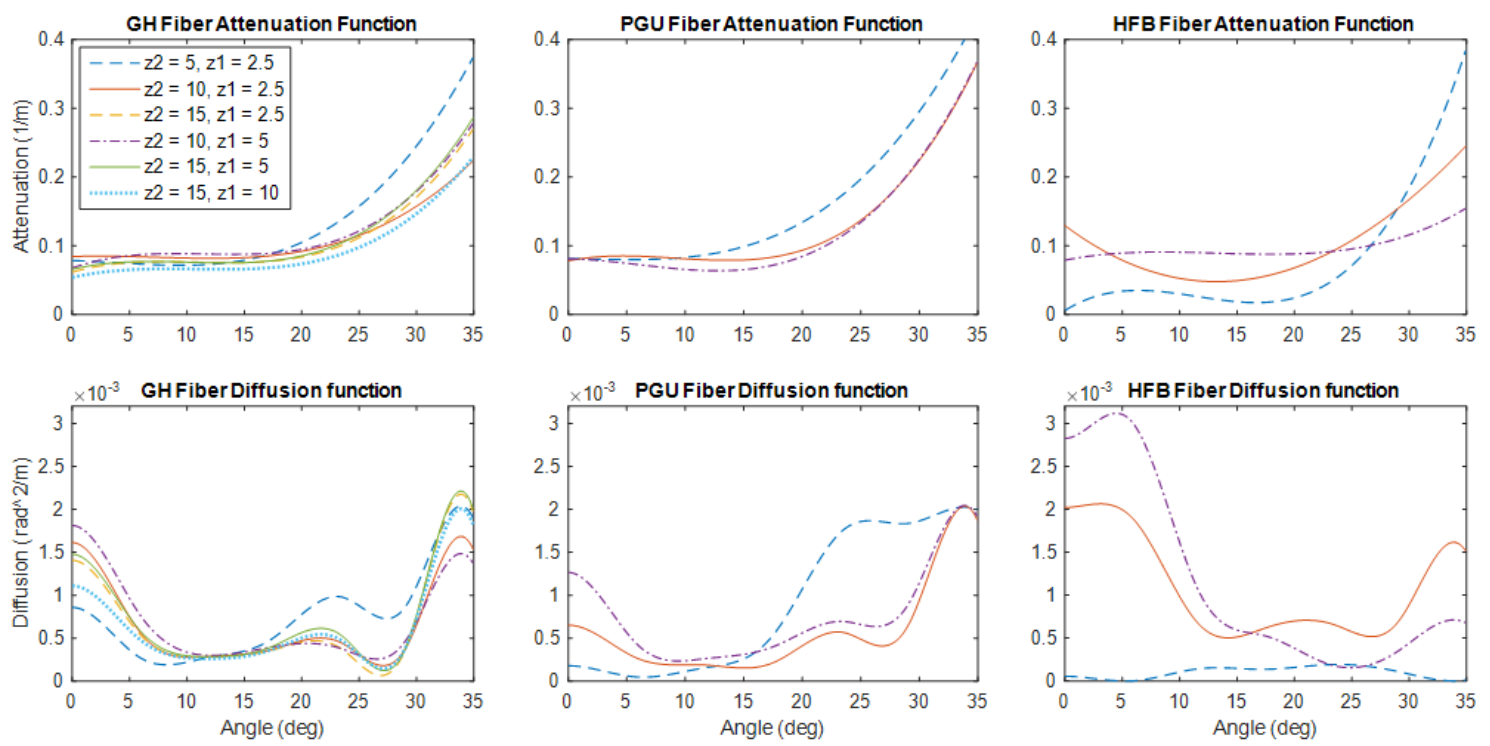

Figure 3: Fitting results of $\boldsymbol{\alpha}(\boldsymbol{\theta})$ and $\boldsymbol{d}(\boldsymbol{\theta})$ for the GH, HFB and $P G U$ fiber.

\section{CONCLUSIONS}

The fitting method detailed in this paper is a convenient tool to explore the possibilities of the developed matrix approach for a new POF model for short fiber lengths that will assist in simulating entire POF systems focusing on the avionics sector. With this method it is possible to create look up tables for the $\alpha(\theta)$ and $d(\theta)$ functions of a particular fiber. Due to the apparent dependency of these functions with the fiber length, these look up tables must provide values for different $z_{1}$ and $z_{2}$ combinations. Our future work will revolve around the expansion of this process to other components in the system, like connectors, further facilitating the simulation of a whole avionics communication system using the two-step procedure detailed in [2].

\section{ACKNOWLEDGEMENTS}

This work has been partly funded by the Fondo Europeo de Desarrollo Regional (FEDER) and by the Spanish Ministerio de Economía y Competitividad under project TEC2015-63826-C3-3-R (MINECO/FEDER). The authors would also like to acknowledge the many useful discussions and technology knowledge gained from the interactions with T. Kien Truong of The Boeing Company. Profs. N. Antoniades and D. Richards also like to acknowledge the travel support from the National Science Foundation (NSF) via award 1633556 entitled USSpain Planning Visit: Plastic Optical Fiber (POF) Systems for Avionics Applications.

\section{REFERENCES}

[1] M. A. Losada, J. Mateo: Short range (in-building) systems and networks: A chance for plastic optical fibers, in WDM Systems and Networks: Modeling, Simulation, Design and Engineering, N. Antoniades, et al., Ed., New York: Springer, 2011.

[2] D. H. Richards et al.: Modeling Methodology for Engineering SI-POF and Connectors in an Avionics System, J. Lightwave Technology, vol. 31, pp. 468-475, Feb. 2013.

[3] D. Gloge. Optical power flow in multimode fibers: Bell Syst Tech. J., vol. 51, n. 8, pp. 1767-1783. 1972.

[4] J. Mateo et al.: Global characterization of optical power propagation in step-index plastic optical fibers, $J$. Opt. Express, vol. 14, pp. 9028-9035, Oct. 2006.

[5] A. Djordjevich, S. Savovic: Numerical Solution of the Power Flow Equation in Step-Index Plastic Optical Fibers, J. Optical Society of America, vol. 21, pp. 1437-1442, Aug. 2004.

[6] F. Breyer et al.: Getting the Impulse Response of SI-POF by Solving the Time-Dependent Power-Flow Equation using the Crank-Nicholson Scheme, in POF Modelling: Theory, Measurement and Application, C. A. Bunge, H. Poisel, Ed., Norderstedt: Verlag Books on Demand GmbH, 2007.

[7] J. Mateo et al.: Frequency response in step index plastic optical fibers obtained from the generalized power flow equation, J. Opt. Express, vol. 17, pp. 2850-2860, Feb. 2009.

[8] M. A. Losada et al.: Characterisation of the far field pattern for plastic optical fibres, in Proc. POF 2004, Nuremberg, Germany, September 2004, pp. 458-465.

[9] M. Andrés et al.: Modelling the Effects of Small-Radius Curvatures in POFs, in Proc. POF 2015, Nuremberg, Germany, September 2015. 